Calidad y medioambiente
Quality and Environment 



\title{
Un índice de capacidad de procesos para distribuciones multivariadas no normales de variables correlacionadas y no correlacionadas
}

\author{
Erasmo Rafael Salazar* \\ Universidad de Oriente. Cumaná, Venezuela \\ José Simón Fermín* \\ Instituto Universitario de Tecnología. Cumaná, Venezuela \\ Recibido: 19 de enero del 2017 / Aprobado: 3 de abril del 2017
}

\begin{abstract}
RESUMEN: En el análisis de capacidad multivariado existen muchos aspectos aún no resueltos en torno a algunos índices, como la no normalidad de los datos y si las variables de calidad están correlacionadas o no están correlacionadas. En este trabajo se pretendió proponer la elaboración de un índice de capacidad multivariado (ICPM) que funcione para ambos casos anteriores por medio de ejemplos de datos simulados. Cabe mencionar que se encontró un amplio desempeño del índice propuesto frente a otros índices de capacidad similares.
\end{abstract}

Palabras clave: índice de capacidad de proceso multivariado / índices de capacidad univariado / distribución no normal / métodos estadísticos

\section{A process capacity index for multivariate non-normal distributions of correlated and uncorrelated variables}

AвSTRACT: In the multivariate analysis of capacity there are many aspects, not even resolved yet, around some indexes such as the non-normality of the data and if the variables of quality are correlated or not. In this work we intend to propose the development of a multivariate capacity index (ICPM, for its acronym in Spanish) that works for both previous cases through simulated data examples. It is worth mentioning that we find a wide performance of the proposed index in comparison with other similar capacity indexes.

Keywords: capacity index of multivariate process / univariate capacity indices / non normal distribution / statistical methods

* Correos electrónicos: erasmo10464407@gmail.com, simon.fermin@gmail.com 


\section{INTRODUCCIÓN}

Los estudios de los Índices de Capacidad de Procesos (ICP) se han desarrollado en los campos univariado y multivariado (ICPM), y son estimaciones numéricas de la capacidad del proceso, es decir, dan una idea de cuán capaz es el proceso (a qué nivel cumple con las especificaciones establecidas). Estos estadísticos son muy útiles ya que, aparte de ser sencillos de calcular, no tienen unidades de medida, por lo que permiten comparar distintos procesos. Básicamente, son el cociente entre la amplitud tolerable del proceso (la distancia entre los límites de tolerancia o límites de especificación) y la amplitud real o natural del proceso (habitualmente, la distancia entre los límites de control es de 6 sigma). Algunos de estos estadísticos se definen a partir de la media del proceso o del objetivo. Los índices de capacidad asociados con la variación a corto plazo son $C_{p}, C_{p k}, C P U$, y $C P L$; por otro lado, los asociados con la variación a largo plazo son $P_{p}, P_{p k}, P P U, P P L$ y $C_{p m}$. En la práctica, se suele considerar que 1,33 es el valor mínimo aceptable para un índice de capacidad (cualquier valor por debajo de esta cifra indicaría que, aunque esté bajo control estadístico, el proceso no cumple con las especificaciones deseadas). Dichos índices se usan bajo el supuesto de que el valor de las mediciones de las variables de calidad representa valores de variables aleatorias independientes con distribución normal y que el proceso se encuentra en control estadístico, como lo establece Montgomery (2009); pero si la distribución fundamental es no normal, entonces los enunciados acerca de la fracción no conforme esperada atribuida a un valor particular de $C_{p}$ o $C_{p k}$ pueden generar resultados erróneos. En la industria existen muchos procesos de manufactura en los que la variabilidad del proceso no se puede describir por una distribución normal. Somerville y Montgomery (1996) reportaron que el uso de índices de capacidad de procesos no normales en vez de normales generarán resultados no confiables para este tipo de procesos. Ellos sugirieron que no se debían utilizar las mismas fórmulas para el cálculo de los índices de capacidad de procesos tradicionales debido a que estas producirían resultados engañosos acerca del desempeño del proceso en cuestión. Un método para tratar con datos no normales es transformarlos utilizando alguna función matemática, de manera que los datos resultantes sean normales; por ejemplo, Tang y Than (1999) iniciaron estudios de simulación para las distribuciones no normales diferentes, tales como log-normal, gamma, y Weibull, y llegaron a la conclusión de 
que el método de Box-Cox funcionaba mejor que los procedimientos de Johnson (1949). Box y Cox (1964) presentan una familia de alternativas de utilidad potencial que convierten los datos no normales en normales. Según Chou, Polansky y Mason (1998); Polansky, Chou y Mason (1998); y Pal(2005), la idea es ajustarlos medianteuna distribución no normal, tales como las distribuciones Weibull, gamma o beta, para luego estimar los cuantiles, los cuales se pueden usar para calcular los índices de capacidad de procesos. Por otro lado, suponiendo que la distribución de datos obtenidos del proceso de muestreo no es normal, se usa el método de percentil de Clements para estimar los índices de capacidad para procesos distribuidos no normales. Este método se utiliza para calcular los índices $C_{p}$ y $C_{p k}$ mediante una familia de curvas de Pearson. Consiste en estimarlos mediante una simple modificación de los ICP normales, utilizando los valores de los puntos percentiles de distribuciones no normales y la mediana como medida central, en lugar de la media. Por otro lado, Liu y Chen (2006) introdujeron una modificación al método de Clements al sustituir los percentiles de las familias de distribuciones de Pearson por una distribución Burr XII apropiada. El desempeño de los ICP no normales a través del método de percentil de Clements se puede usar en las distribuciones Weibull, Log-normal, beta, gamma y la Pareto generalizada (DPG2), todas con dos parámetros.

Según Valdiviezo y Fermín (2010), el método de percentil de Clements es propuesto para determinar los ICP y reemplaza en la ecuación del $C_{p}$ el valor $6 \sigma$ por la longitud del intervalo entre el límite superior e inferior de los puntos percentiles 0,135 y 99,865 de una distribución $X$, esto es respectivamente $U_{p}-L_{p}$.

$$
C C_{p}=\frac{U-L}{U_{p}-L_{p}}
$$

Donde $U_{p}$ es el percentil 99,865 y $L_{p}$ es el percentil 0,135.

Para el $C_{p k}$, la media del proceso $\mu$ es estimada por la mediana $M_{d}$ y los valores de $3 \sigma$ son estimados por $M_{d}-L_{p}$ y $U_{p}-M_{d}$, respectivamente. Así, se obtienen las siguientes expresiones:

$$
C C_{p l}=\frac{M_{d}-L}{M_{d}-L_{p}}
$$




$$
\begin{gathered}
C C_{p u}=\frac{U-M_{d}}{U_{p}-M_{d}} \\
C C_{p k}=\min \left\{C C_{p l}, C C_{p u}\right\}
\end{gathered}
$$

Muchos autores realizan investigaciones de ICP considerando una sola variable; sin embargo, es sabido que la gran mayoría de estos estudios abarcan más de una variable. En los estudios de índices de capacidad de dominio multivariado también se han propuesto algunos índices de capacidad que son extensiones de sus contrapartes univariadas, ya que -como lo señalan Foster, Barton y Gautan (2005) - aún no hay una metodología sólida para calcular índices de capacidad multivariados (ICPM) y sobre todo para el caso no normal; por tanto, hasta la fecha no hay consenso sobre el uso de un índice en particular. Además, no existe un ICPM que sea consistente y suficiente para el caso normal y no normal, y para variables correlacionadas y no correlacionadas. De aquí se desprende la necesidad de diseñar un ICPM para evaluar la capacidad de un proceso para los casos anteriores expuestos, sobre todo cuando las variables están correlacionadas. Las investigaciones sobre índices de capacidad multivariados empezaron a principios de 1990, pero no fue sino hasta el 2005 cuando el número de publicaciones se incrementó y desde entonces diferentes ICPM han sido propuestos. Estos ICPM se pueden dividir en cuatro grupos diferentes, como lo sugieren Shinde y Khadse (2009): grupo 1 se basa en la razón de una región de tolerancia y una región del proceso -ver como un ejemplo a Taam y Liddy (1993); Shahriari y Abdollahzadeh (2009); Pan y Lee (2010); y Cuamea y Rodríguez (2014)-. El grupo 2 se basa en la probabilidad de producto de no conformidad -ver como ejemplo a Castagliola y Castellanos (2005)-; el grupo 3 se basa en el análisis de componentes principales -ver como ejemplo a Wang y Chen (1998)-; y un grupo 4 llamado otros (Shahriari y Lawrence, 1995).

Los ICPM, en general pueden ser obtenidos de tres maneras: (a) desde la proporción de una región de tolerancia a una región de proceso, (b) calculando la probabilidad de productos que no cumplen con las reglas y (c) por los enfoques en que las funciones de pérdida son empleadas. Ahora, de forma adicional se plantea la propuesta de este trabajo de investigación, que se basa en el siguiente enunciado de Cuamea y Anaya (2009): "En el estudio multivariado, las variables críticas deben 
estar dentro de sus respectivas especificaciones para que el producto se considere aceptable. Si solo una de ellas no cumple con las especificaciones esto será suficiente para que el cliente considere inaceptable el producto". De acuerdo con esto, para el caso multivariado se hace el estudio univariado a cada variable de datos no normales y se concluye, con respecto al caso multivariado no normal, usando un índice propuesto en este trabajo.

\section{2. ÍNDICE DE CAPACIDAD DE PROCESOS MULTIVARIADO}

Hubele, Shahiari y Cheng (1991) usaron la distribución multivariada normal para definir un ICPM como la razón de una región de tolerancia rectangular y una región de tolerancia modificada, que es el rectángulo más pequeño alrededor de la elipse con un error especificado de tipo I ( $\alpha=0.0027)$. El número de las variables de calidad en el proceso es tenido en cuenta tomando la $v$ ésima raíz de la proporción, donde $v$ representa el número de las variables de calidad.

$$
C_{P M}=\sqrt[v]{\frac{\text { volumen de la región de tolerancia de ingeniería }}{\text { volumen de la región de procesos de ingeniería }}}
$$

Taam et al. (1993) definieron un índice de capacidad multivariado $\left(M C_{P M}\right)$ como la razón del volumen de la región de tolerancia modificada y el volumen de la escala 99,73 \% de la región de proceso. La región de tolerancia modificada es definida como el elipsoide más grande centrado en el blanco totalmente ubicado dentro de la región de tolerancia original. Luego, para ajustar el cálculo estimado del índice de capacidad normal multivariado, usaron lo siguiente:

$$
\widehat{M C}_{P M}=\frac{C_{P M}}{\hat{D}}
$$

Donde $\hat{D}=\sqrt{1+\frac{n}{n+1}\left(\overline{\boldsymbol{X}}-\mu_{0}\right) \boldsymbol{S}^{-1}\left(\overline{\boldsymbol{X}}-\mu_{0}\right)^{T}} ; \overline{\boldsymbol{X}}$ es el vector media muestral, $\boldsymbol{S}$ es la matriz de varianza-covarianza muestral, y $\boldsymbol{\mu}_{0}$ es la media objetivo del proceso.

Wang, Hubele, Lawrence, Miskulin y Shahriari (2000) compararon los ICPM mencionados anteriormente y presentaron algunos ejemplos 
gráficos para ilustrarlos. Sin embargo, la correlación entre las variables no fue tenida en cuenta. Ellos estimaron tanto en conjunto como individuales los índices de capacidad de proceso para los datos multivariado (sin la correlación). Castagliola y Castellanos (2005) prolongaron el método univariado de Castagliola a la distribución multivariada, reemplazando la función de densidad de probabilidad univariado $f(x)$ por su versión multivariada. Mientras que Wang et al. (2000) y Wang y Du (2000) propusieron un método en el que se utiliza el análisis de componentes principales para describir el comportamiento de un proceso para datos multivariados. La ventaja de esta técnica radica en que es capaz no solo de reducir la dimensión del problema, sino también de transformar datos correlacionados en independientes, lo cual simplifica considerablemente los cálculos. Cuamea y Anaya (2009) establecieron los índices $C_{p M}$ y $C_{p k M}$ para una distribución normal multivariada para variables múltiples con correlación, sin embargo, $C_{p M}$ puede usarse para variables sin correlación.

Wang (2006) también usó la distancia geométrica en procesos no normales y normales, multivariados. Yeh y Chen (2001) aplicaron métodos no paramétricos para calcular proporciones no ajustadas e índice de capacidad de proceso multivariado para procesos no normales.

\section{MATERIALES Y MÉTODOS}

Dado un conjunto de $v$ variables que resultan de un proceso aleatorio (Monte Carlo) de muestreo, se obtienen un conjunto de datos de diferentes tamaños, modelados por una distribución no normal cualquiera, de la cual se obtienen las estimaciones de los parámetros para determinar el ICPM que va a establecer la capacidad del proceso de producción para cada muestra. Un requisito es que se debe contar con especificaciones para cada una de las $v$ variables de calidad y con estimaciones de los parámetros del proceso, tales como un vector de medias y una matriz de varianzas covarianzas.

Para el caso univariado, se tienen los límites de especificación inferior y superior para el control de la variable de calidad $X$; el objetivo que se persigue es que ( $L E I \leq X \leq L E S)$, donde $L E I$ y $L E S$ son los límites de especificación inferior y superior establecidos para la variable $X$. Para el caso multivariado, lo anterior se extiende y el intervalo es reemplazado por una región de especificación; dicha región de especificación estará construida por los intervalos de especificación para cada 
una de las variables de calidad. La región de especificación sería representada mediante las desigualdades:

$$
I_{i=1}^{v}\left(L E I_{i} \leq X_{i} \leq L E S_{i}\right)
$$

Ahora, para el caso no normal multivariado, en este artículo se planteó la siguiente propuesta en estudio: sean $X_{1}, X_{2}, \ldots, X_{v}, v$ variables aleatorias obtenidas de un proceso, modeladas por una función de distribución multivariado no normal, de donde se obtienen las estimaciones de los parámetros para el cálculo de cada ICP para cada $X_{i}, i=1, \ldots, v$. Después de obtener cada ICP para cada $X_{i}, i=1, \ldots, v$, se procede a determinar el índice multivariado CPME .

Se usarán varias muestras de diferentes tamaños, donde las variables estarán correlacionadas y no correlacionadas, para que a través del modelo obtenido se realicen comparaciones de los resultados de los métodos multivariados empleados por otros autores. Para esto, el paquete estadístico Statgraphics usa el ICPM, $M C P=\frac{Z}{K / 2}$, donde $Z$ es el valor de la variable aleatoria normal estándar correspondiente a los DPM calculados. Donde el DPM es el índice de capacidad defecto por millón que representa el número estimado de las muestras por millón que estarían fuera de la especificación en una o más variables. $K$ es el múltiplo del sigma y en general $K=6$. MCP se interpreta similarmente a $C_{p k}$ caso univariado, así un $M C P \geq 1,33$ es el deseado. Además, el paquete estadístico Minitab V. 17 calcula los índices de capacidad de procesos para el caso no normal $P P, P P l, P P u$ y $P p_{k}$. Otros índices calculados se determinarán usando el software Matlab V. 7.6.0, Excel y el paquete estadístico SPSS V. 15.0 para Windows.

\section{PROPOSICIÓN DE ÍNDICE DE CAPACIDAD PARA USO EN CASO DE VARIABLES MÚLTIPLES (CPME)}

Dado un conjunto de $v$ variables que resultan de un proceso de muestreo, se obtiene un conjunto de datos modelados por una distribución normal o no normal, de la cual se obtienen las estimaciones de los parámetros para determinar el ICPM que va a definir la capacidad del proceso de producción. Cuando los datos no son normales, entonces el índice multivariado requiere una nueva metodología. En función de lo expuesto, en este trabajo se evaluó el desempeño de los métodos de 
estimación de capacidad que existen para datos con variables de calidad multivariados no normal.

Sea $X=\left(X_{1}, X_{2}, \ldots, X_{v}\right)^{\prime}$ el vector de las $v$ variables de calidad del proceso.

Sea $\mu=\left(\mu_{1}, \mu_{2}, \ldots, \mu_{v}\right)^{\prime}$ el vector de medias, y $\sum$ su matriz de covarianzas.

Sea $S=\left\{X \in \mathbb{R}^{v}: L E I_{i} \leq X \leq L E S_{i}\right\} i=1, \ldots, v$, la región de tolerancias del proceso.

Se obtienen las estimaciones de los parámetros para el cálculo de cada ICP para cada $X_{i}, i=1, \ldots, v$. Se propone el ICPM definido de la siguiente manera:

$$
\chi_{A_{i}}\left(I c p_{i}\right)=\left\{\begin{array}{ccc}
1 & \text { si } & I c p_{i} \in A_{i} \\
0 & \text { si } & I c p_{i} \notin A_{i}
\end{array}\right.
$$

Donde $A_{i}=\operatorname{Rango}\left(\operatorname{Icp}_{i}\right)=\left\{I_{c p_{i}} / \operatorname{Icp}_{i} \geq 1,33\right\}$ para cada $X_{i}, i=1, \ldots, v$ y $I c p_{i}$ : es un índice de capacidad univariado seleccionado. $I c p=C C_{p}$ es adecuado para el caso no normal.

Se define el siguiente ICPM CPME $=\frac{\sum_{i=1}^{v} \chi_{A_{i}}\left(I c p_{i}\right)}{v}$

Donde, CPME significa Capacidad de Proceso Multivariado Estadístico.

Ahora bien, por definición de CPME se tiene que:

Si $\mathrm{CPME}=1$, entonces el proceso de producción es estable, ya que todas las variables están en el rango de especificación dado.

Si $\mathrm{CPME} \neq 1$, entonces el proceso de producción no es estable, ya que existe al menos una $X_{i}, i=1, \ldots, v$ que está fuera del rango de especificación dado para cada $X_{i}, i=1, \ldots, v$. 


\section{RESULTADOS}

En las siguientes secciones se muestra la obtención del índice de capacidad propuesto en este trabajo para variables no normales con correlación y sin correlación, así como la de otros índices.

\subsection{Cálculo de CPME y comparación con otros índices propuestos}

En este trabajo se usan varios casos de muestras diferentes de datos no normales simulados, obtenidos a través del método de Monte Carlo y se trabaja con dos: variables correlacionadas y no correlacionadas. En ellos se calculan índices univariados y luego el índice multivariado propuesto en este trabajo y se comparan con otros índices obtenidos. Además, para que el estudio sea eficiente y suficiente, se generan aleatoriamente las variables $C_{1}$ y $C_{2}$ no normales para los seis tamaños diferentes de muestras $n=10,20,50,100,1000$ y 10000 . Se analizará este caso de manera univariante y bivariante, usando los paquetes estadísticos Minitab v. 17 para el caso univariante y Statgraphics Centurion para el caso multivariante. Sin embargo, ya que las variables son no normales, se usará la técnica aplicada de Box-Cox para el cálculo de los índices multivariados de Cuamea y Rodríguez (2014), MCP y de Taam et. al. (1993). Así, se obtienen las siguientes tablas de resultados: 


\subsection{Caso variables correlacionadas}

Tabla 1

Resumen descriptivo para las variables no normales $C_{1}$ y $C_{2}$ para diferentes tamaños de muestras

\begin{tabular}{|c|c|c|c|c|c|}
\hline \multirow{2}{*}{$\begin{array}{l}\text { Muestra } \\
\text { Variable }\end{array}$} & \multicolumn{5}{|c|}{$n=10$} \\
\hline & LEI & LES & Objetivo & Media & Desv. est. (general) \\
\hline $\mathrm{C}_{1}$ & 0,2 & 3,2 & 1,2 & 1,17 & 1,1 \\
\hline $\mathrm{C}_{2}$ & 0,05 & 10,3 & 5,0 & 3,6 & 3,4 \\
\hline Muestra & & \multicolumn{4}{|c|}{$n=20$} \\
\hline Variable & LEI & LES & Objetivo & Media & Desv. est. (general) \\
\hline $\mathrm{C}_{1}$ & 0,01 & 3,6 & 1,2 & 0,9 & 0,97 \\
\hline $\mathrm{C}_{2}$ & 0,2 & 20,0 & 5,0 & 5,9 & 5,6 \\
\hline Muestra & & \multicolumn{4}{|c|}{$n=50$} \\
\hline Variable & LEI & LES & Objetivo & Media & Desv. est. (general) \\
\hline$C_{1}$ & 0,01 & 2,9 & 1,2 & 0,99 & 0,79 \\
\hline $\mathrm{C}_{2}$ & 0,17 & 21,9 & 5,0 & 4,8 & 4,81 \\
\hline Muestra & & \multicolumn{4}{|c|}{$\boldsymbol{n}=100$} \\
\hline Variable & LEI & LES & Objetivo & Media & Desv. est. (general) \\
\hline $\mathrm{C}_{1}$ & 0,05 & 7,2 & 1,2 & 1,1 & 1,18 \\
\hline $\mathrm{C}_{2}$ & 0,17 & 29,0 & 5,0 & 6,6 & 6,7 \\
\hline Muestra & & \multicolumn{4}{|c|}{$n=1000$} \\
\hline Variable & LEI & LES & Objetivo & Media & Desv. est. (general) \\
\hline $\mathrm{C}_{1}$ & 0,00008 & 7,0 & 1,2 & 1,07 & 1,07 \\
\hline $\mathrm{C}_{2}$ & 0,0017 & 38,0 & 5,0 & 5,2 & 5,25 \\
\hline Muestra & & \multicolumn{4}{|c|}{$n=10000$} \\
\hline Variable & LEI & LES & Objetivo & Media & Desv. est. (general) \\
\hline $\mathrm{C}_{1}$ & 0,00001 & 9,7 & 1,2 & 1,01 & 1,01 \\
\hline $\mathrm{C}_{2}$ & 0,0002 & 44,0 & 5,0 & 5,02 & 5,01 \\
\hline
\end{tabular}

Elaboración propia 
Tabla 2

ICP univariado de las variables no normal $C_{1}$ y $C_{2}$ para diferentes tamaños de muestras

\section{Tamaño de muestra}

\begin{tabular}{|c|c|c|c|c|c|c|c|c|c|c|c|c|}
\hline & \multicolumn{2}{|c|}{$n=10$} & \multicolumn{2}{|c|}{$n=20$} & \multicolumn{2}{|c|}{$n=50$} & \multicolumn{2}{|c|}{$n=100$} & \multicolumn{2}{|c|}{$n=1000$} & \multicolumn{2}{|c|}{$\boldsymbol{n}=10000$} \\
\hline Variables & $C_{1}$ & $C_{2}$ & $C_{1}$ & $C_{2}$ & $C_{1}$ & $C_{2}$ & $C_{1}$ & $C_{2}$ & $C_{1}$ & $C_{2}$ & $C_{1}$ & $C_{2}$ \\
\hline \multicolumn{13}{|l|}{$\begin{array}{l}\text { Índice de } \\
\text { capacidad }\end{array}$} \\
\hline$P P$ & 0,49 & 0,29 & 0,57 & 0,51 & 1,39 & 0,93 & 1,08 & 0,69 & 0,96 & 1,06 & 1,44 & 1,32 \\
\hline$P P l$ & 0,79 & 0,98 & 0,99 & 0,95 & 0,94 & 0,95 & 0,94 & 0,97 & 1,00 & 1,00 & 1,00 & 1,00 \\
\hline$P P u$ & 0,44 & 0,25 & 0,52 & 0,46 & 1,47 & 0,71 & 1,10 & 0,65 & 0,96 & 1,06 & 1,49 & 1,25 \\
\hline$P p_{k}$ & 0,44 & 0,25 & 0,52 & 0,46 & 0,94 & 0,71 & 0,94 & 0,65 & 0,96 & 1,00 & 1,00 & 1,00 \\
\hline$C C p$ & 1,00 & 0,99 & 0,98 & 1,00 & 0,98 & 1,00 & 1,00 & 1,00 & 1,00 & 1,00 & 1,44 & 1,28 \\
\hline$C C p k$ & 0,98 & 0,99 & 0,98 & 0,99 & 0,98 & 1,00 & 1,00 & 1,00 & 1,00 & 0,98 & 1,00 & 1,00 \\
\hline $\begin{array}{l}\text { Correlación } \\
\text { de Pearson }\end{array}$ & \multicolumn{2}{|c|}{0,904} & \multicolumn{2}{|c|}{0,982} & \multicolumn{2}{|c|}{0,953} & \multicolumn{2}{|c|}{0,951} & \multicolumn{2}{|c|}{0,997} & \multicolumn{2}{|c|}{1,00} \\
\hline
\end{tabular}

Elaboración propia

Para el caso multivariado, los ICPM son los siguientes:

Tabla 3

Cálculo de ICPM para diferentes tamaños de muestra respecto a la variable $C=\left(C_{1}, C_{2}\right)$ no normal

\begin{tabular}{|c|c|c|c|c|c|c|c|}
\hline & $\begin{array}{c}\text { Índice de } \\
\text { capacidad } \\
\text { multivariado }\end{array}$ & $n=10$ & $n=20$ & $n=50$ & $n=100$ & $n=1000$ & $n=10000$ \\
\hline $\begin{array}{l}\text { Cuamea y } \\
\text { Rodríguez (2014) }\end{array}$ & СpM & 0,44 & 0,44 & 0,57 & 0,6 & 21,5 & 82,06 \\
\hline $\begin{array}{l}\text { Statgraphics C. } \\
(2015)\end{array}$ & $M C P$ & 0,35 & 0,42 & 0,52 & 0,60 & 0,78 & 0,86 \\
\hline Taam et al. (1993) & $M C p$ & 3,8 & 5,7 & 6,8 & 4,15 & 12,3 & $\infty$ \\
\hline $\begin{array}{l}\text { Índice propuesto } \\
\text { en esta } \\
\text { investigación }\end{array}$ & $C P M E(C C p)$ & $\neq 1$ & $\neq 1$ & $\neq 1$ & $\neq 1$ & $\neq 1$ & $\neq 1$ \\
\hline
\end{tabular}

Elaboración propia 
Los gráficos de tolerancia para cada caso muestral se observan en la figura 1 .

Gráfico de tolerancia para $C_{1}$ y $C_{2}$. Variables correlacionadas $n=10$

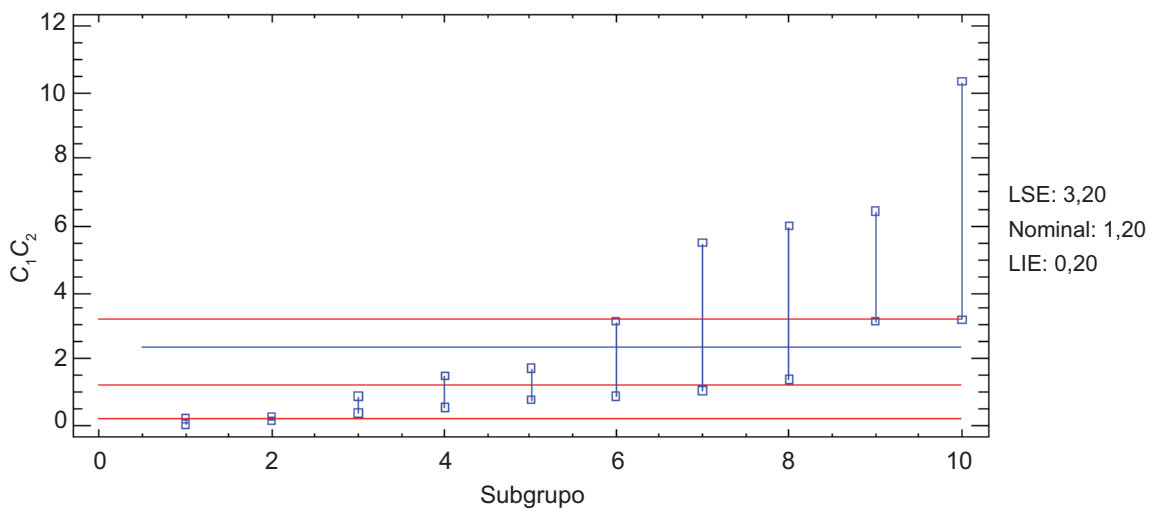

Gráfico de tolerancia para $C_{1}$ y $C_{2}$. Variables correlacionadas $n=20$

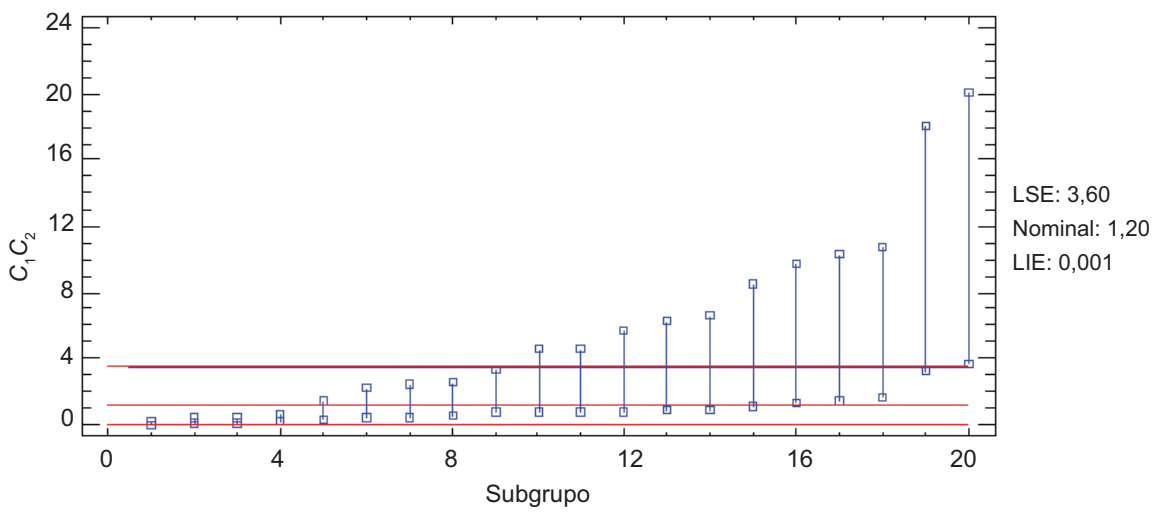

Gráfico de tolerancia para $C_{1}$ y $C_{2}$. Variables correlacionadas $n=50$

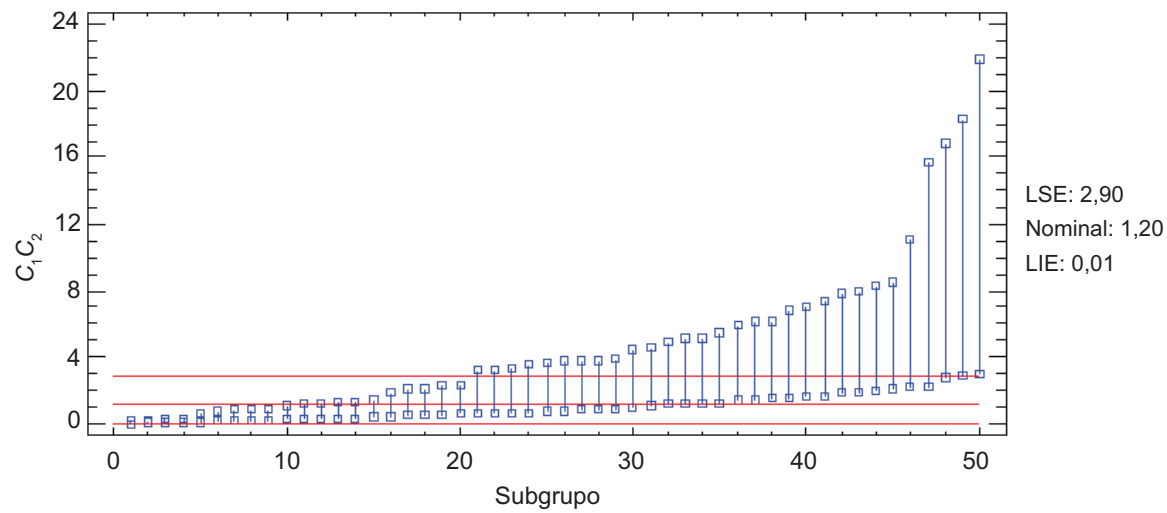


Un índice de capacidad de procesos para distribuciones multivariadas no normales

Gráfico de tolerancia para $C_{1}$ y $C_{2}$. Variables correlacionadas $n=100$

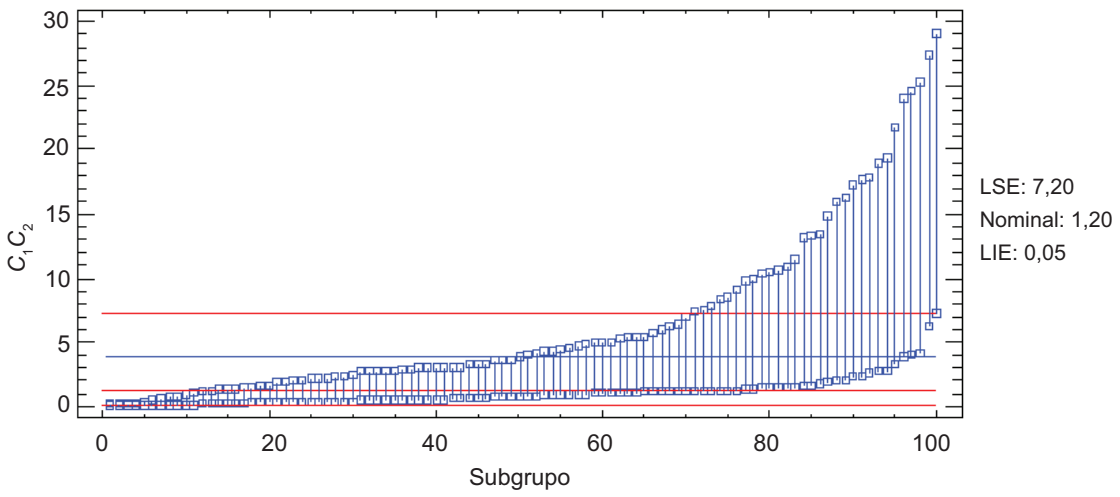

Gráfico de tolerancia para $C_{1}$ y $C_{2}$. Variables correlacionadas $n=1000$

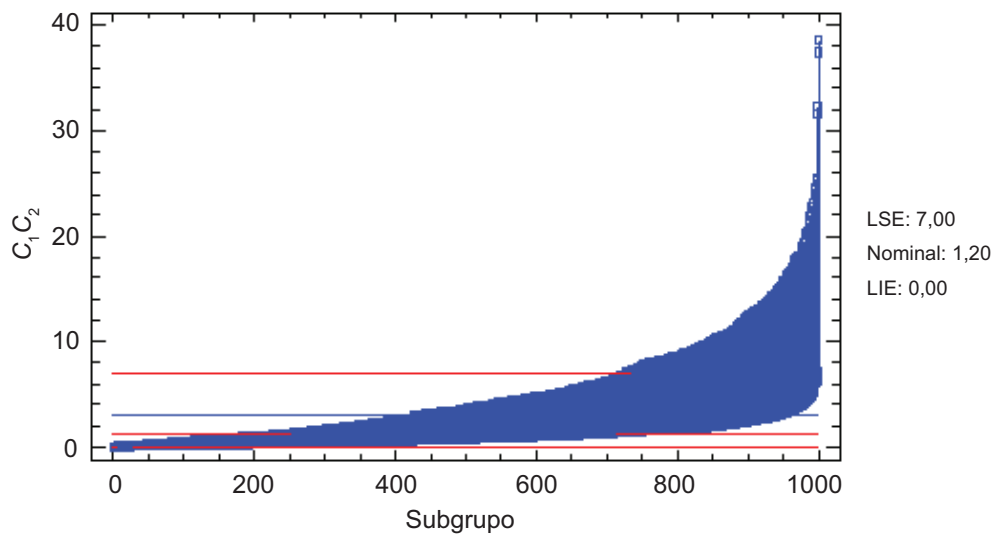

Gráfico de tolerancia para $C_{1}$ y $C_{2}$. Variables correlacionadas $n=10000$

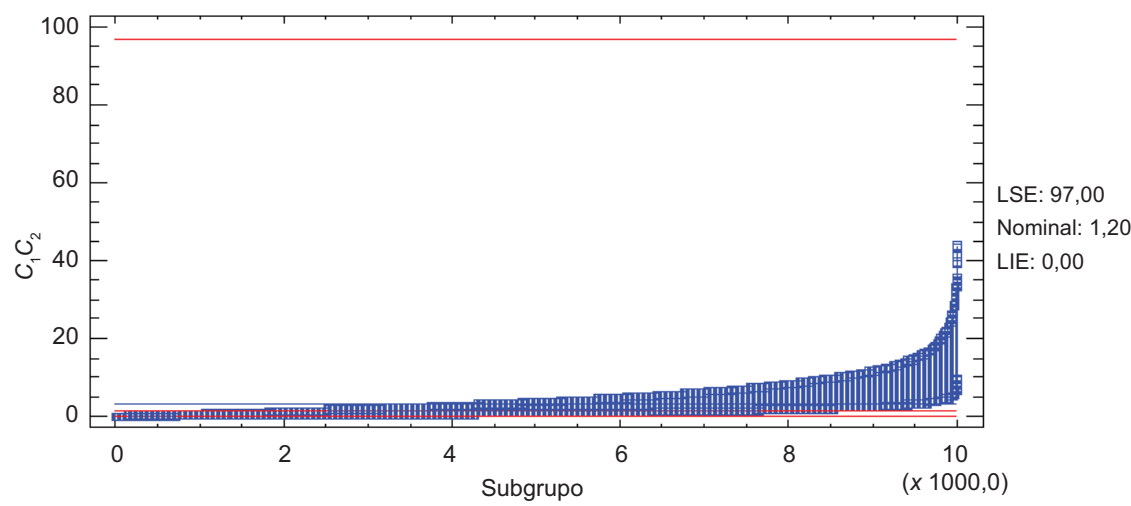

Figura 1. Gráficos de tolerancia para las variables $\mathrm{C}_{1}, \mathrm{C}_{2}$. Caso variables correlacionadas. $n=10 ; 20 ; 50 ; 100 ; 1000$ y 10000

Elaboración propia 


\subsection{Caso de variables no correlacionadas}

Para estudio del caso de variables no correlacionadas, los resultados son los siguientes:

Tabla 4

Resumen descriptivo de las variables no normales $C_{1}$ y $C_{2}$ para diferentes tamaños de muestras

\begin{tabular}{|c|c|c|c|c|c|}
\hline \multirow{2}{*}{$\begin{array}{l}\text { Muestra } \\
\text { Variable }\end{array}$} & \multicolumn{5}{|c|}{$n=10$} \\
\hline & LEI & LES & Objetivo & Media & Desv. est. (general) \\
\hline$C_{1}$ & 0,04 & 1,2 & 0,4 & 0,51 & 0,408 \\
\hline $\mathrm{C}_{2}$ & 0,05 & 13,0 & 6,0 & 4,63 & 4,235 \\
\hline Muestra & \multicolumn{5}{|c|}{$n=20$} \\
\hline Variable & LEI & LES & Objetivo & Media & Desv. est. (general) \\
\hline$C_{1}$ & 0,02 & 3,7 & 0,4 & 0,954 & 0,999 \\
\hline $\mathrm{C}_{2}$ & 0,25 & 21,1 & 6,0 & 6,116 & 6,186 \\
\hline Muestra & \multicolumn{5}{|c|}{$n=50$} \\
\hline Variable & LEI & LES & Objetivo & Media & Desv. est. (general) \\
\hline$C_{1}$ & 0,08 & 3,6 & 0,4 & 1,09 & 0,963 \\
\hline $\mathrm{C}_{2}$ & 0,02 & 19,9 & 6,0 & 4,71 & 4,431 \\
\hline Muestra & \multicolumn{5}{|c|}{$n=100$} \\
\hline Variable & LEI & LES & Objetivo & Media & Desv. est. (general) \\
\hline $\mathrm{C}_{1}$ & 0,01 & 7,2 & 0,4 & 1,04 & 1,164 \\
\hline $\mathrm{C}_{2}$ & 0,004 & 22,8 & 6,0 & 5,02 & 4,857 \\
\hline Muestra & \multicolumn{5}{|c|}{$n=1000$} \\
\hline Variable & LEI & LES & Objetivo & Media & Desv. est. (general) \\
\hline$C_{1}$ & 0,001 & 7,0 & 0,4 & 1,032 & 1,041 \\
\hline $\mathrm{C}_{2}$ & 0,008 & 22,8 & 6,0 & 4,956 & 4,910 \\
\hline Muestra & \multicolumn{5}{|c|}{$n=10000$} \\
\hline Variable & LEI & LES & Objetivo & Media & Desv. est. (general) \\
\hline$C_{1}$ & 0,04 & 4,0 & 0,4 & 1,3333 & 0,5095 \\
\hline $\mathrm{C}_{2}$ & 0,16 & 3,2 & 1,7 & 0,5695 & 0,4781 \\
\hline
\end{tabular}

Elaboración propia 
Tabla 5

ICP univariado de las variables no normales $C_{1}$ y $C_{2}$ para diferentes tamaños de muestras

\begin{tabular}{lccccccccccccc}
\hline & \multicolumn{10}{c}{ Tamaño de muestra } \\
\cline { 2 - 5 } & \multicolumn{1}{c}{$\boldsymbol{n}=10$} & \multicolumn{1}{c}{$\boldsymbol{n}=50$} & $\boldsymbol{n}=100$ & $\boldsymbol{n}=1000$ & $\boldsymbol{n}=10000$ \\
\hline Variables & $\boldsymbol{C}_{1}$ & $\boldsymbol{C}_{2}$ & $\boldsymbol{C}_{1}$ & $\boldsymbol{C}_{2}$ & $\boldsymbol{C}_{1}$ & $\boldsymbol{C}_{2}$ & $\boldsymbol{C}_{1}$ & $\boldsymbol{C}_{2}$ & $\boldsymbol{C}_{1}$ & $\boldsymbol{C}_{2}$ & $\boldsymbol{C}_{1}$ & $\boldsymbol{C}_{2}$ \\
\hline $\begin{array}{l}\text { Índice de } \\
\text { capacidad }\end{array}$ & & & & & & & & & & & & \\
\hline$P P$ & 0,42 & 0,38 & 0,58 & 0,52 & 0,62 & 0,70 & 0,88 & 0,66 & 1,03 & 0,7 & 1,28 & 1,15 \\
$P P l$ & 0,90 & 0,98 & 0,97 & 0,94 & 0,91 & 1,00 & 0,99 & 1,00 & 1,00 & 1,00 & 1,06 & 1,12 \\
$P P u$ & 0,34 & 0,32 & 0,49 & 0,47 & 0,57 & 0,66 & 0,88 & 0,63 & 1,03 & 0,67 & 1,43 & 1,18 \\
$P p_{k}$ & 0,34 & 0,32 & 0,49 & 0,47 & 0,57 & 0,66 & 0,88 & 0,63 & 1,00 & 0,67 & 1,06 & 1,12 \\
$C C p$ & 1,00 & 0,99 & 0,98 & 1,00 & 0,98 & 1,00 & 2,00 & 1,14 & 1,00 & 0,7 & 1,40 & 1,38 \\
$C C p k$ & 0,77 & 0,99 & 0,97 & 1,00 & 0,98 & 1,00 & 1,09 & 1,00 & 1,00 & 0,66 & 1,04 & 1,12 \\
\hline $\begin{array}{l}\text { Correlación } \\
\text { de Pearson }\end{array}$ & $-0,22$ & $-0,174$ & $-0,229$ & $-0,055$ & $-0,025$ & 0,003 \\
\hline
\end{tabular}

Elaboración propia

Para el caso multivariado, los ICPM son los siguientes:

Tabla 6

Cálculo de ICPM para diferentes tamaños de muestra respecto de la variable $C=\left(C_{1}, C_{2}\right)$ no normal

\begin{tabular}{|c|c|c|c|c|c|c|c|}
\hline & $\begin{array}{c}\text { Índice de } \\
\text { capacidad } \\
\text { multivariado }\end{array}$ & $n=10$ & $n=20$ & $n=50$ & $\boldsymbol{n}=100$ & $\boldsymbol{n}=1000$ & $\boldsymbol{n}=10000$ \\
\hline $\begin{array}{l}\text { Cuamea y } \\
\text { Rodríguez (2014) }\end{array}$ & CpM & 0,40 & 0,67 & 0,71 & 0,67 & 0,87 & 0,96 \\
\hline $\begin{array}{l}\text { Statgraphics C. } \\
(2015)\end{array}$ & $M C P$ & 0,23 & 0,35 & 0,44 & 0,60 & 0,79 & 1,00 \\
\hline $\begin{array}{l}\text { Taam et al. } \\
\text { (1993) }\end{array}$ & $M C p$ & 1,1 & 1,00 & 1,03 & 1,00 & 1,00 & 1,00 \\
\hline $\begin{array}{l}\text { Índice de esta } \\
\text { investigación }\end{array}$ & $C P M E(C C p)$ & $\neq 1$ & $\neq 1$ & $\neq 1$ & $\neq 1$ & $\neq 1$ & 1 \\
\hline
\end{tabular}

Elaboración propia 
Los gráficos de tolerancia para cada muestra se observan en la figura 2.

Gráfico de tolerancia para $C_{1}$ y $C_{2}$. Variables no correlacionadas $n=10$

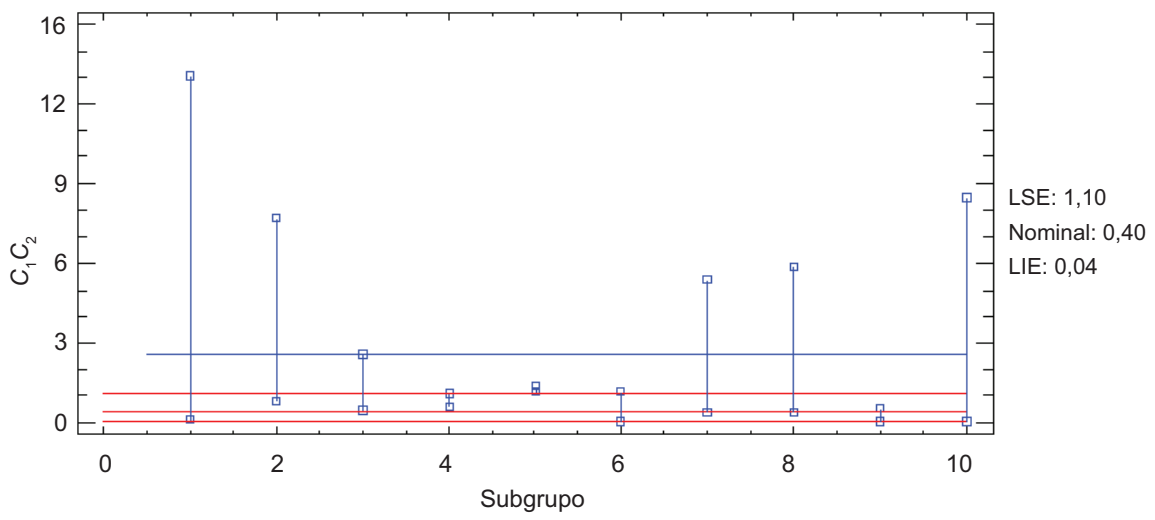

Gráfico de tolerancia para $C_{1}$ y $C_{2}$. Variables no correlacionadas $n=20$

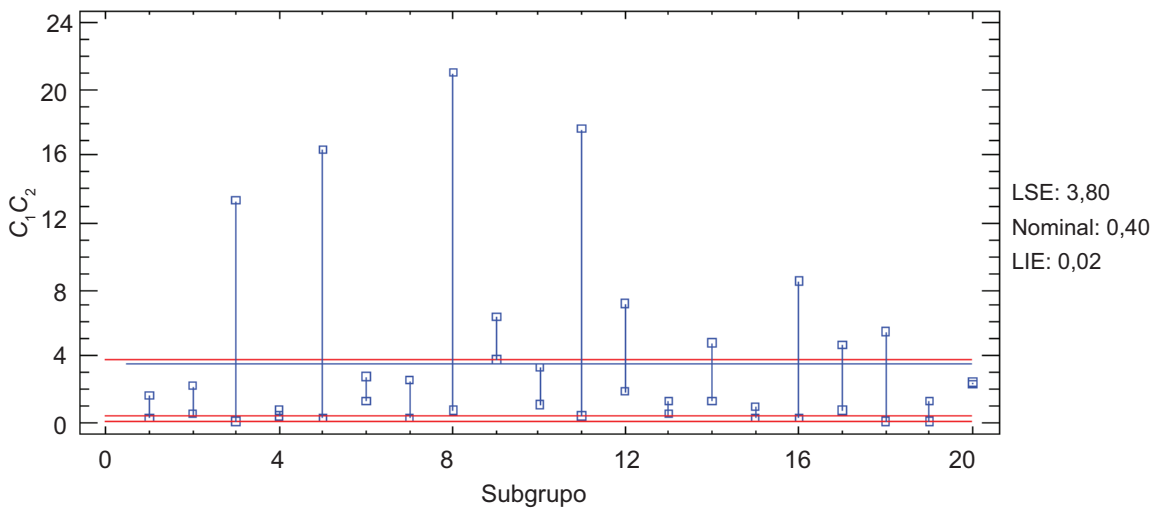

Gráfico de tolerancia para $C_{1}$ y $C_{2}$. Variables no correlacionadas $n=50$

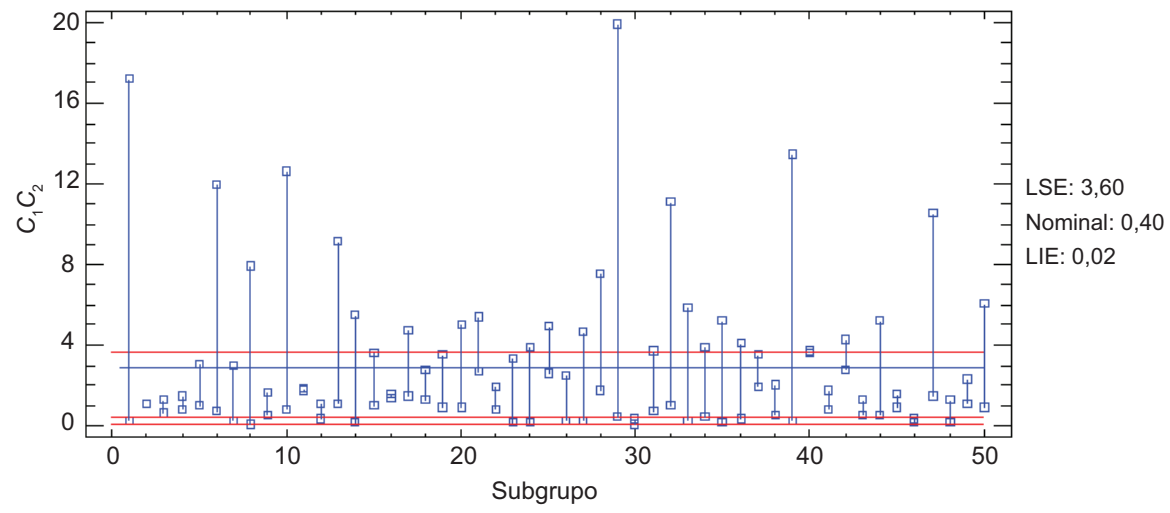




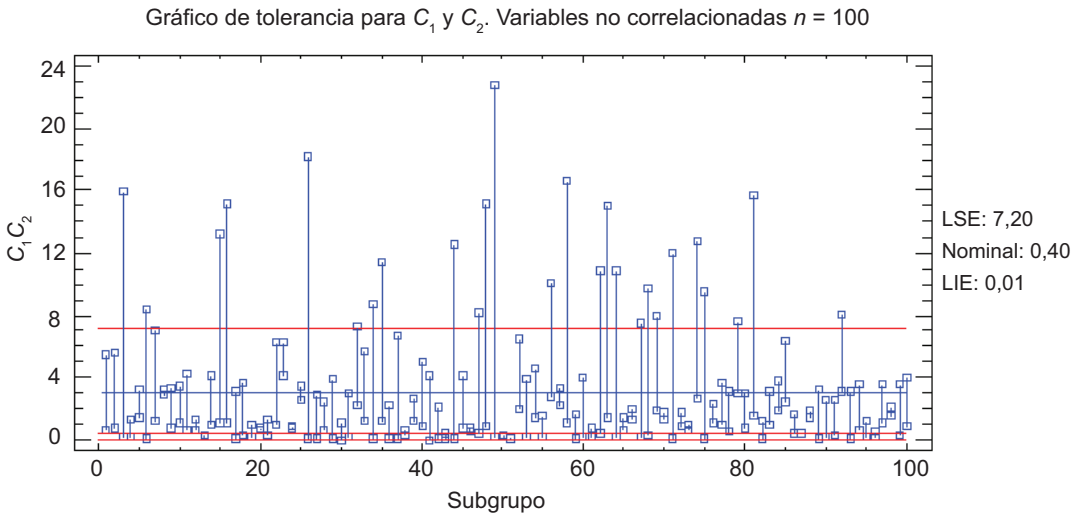

Gráfico de tolerancia para $C_{1}$ y $C_{2}$. Variables no correlacionadas $n=1000$

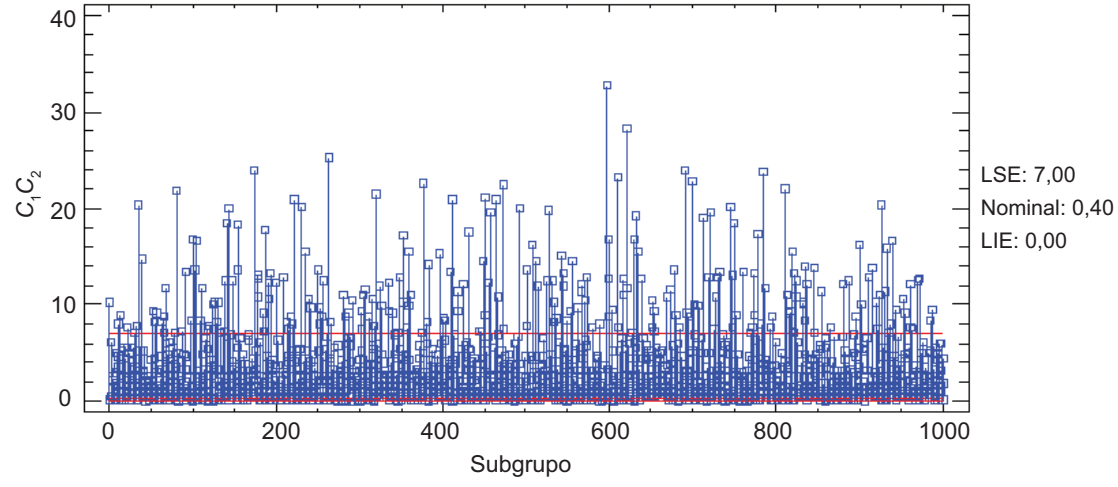

Gráfico de tolerancia para $C_{1}$ y $C_{2}$. Variables no correlacionadas $n=10000$

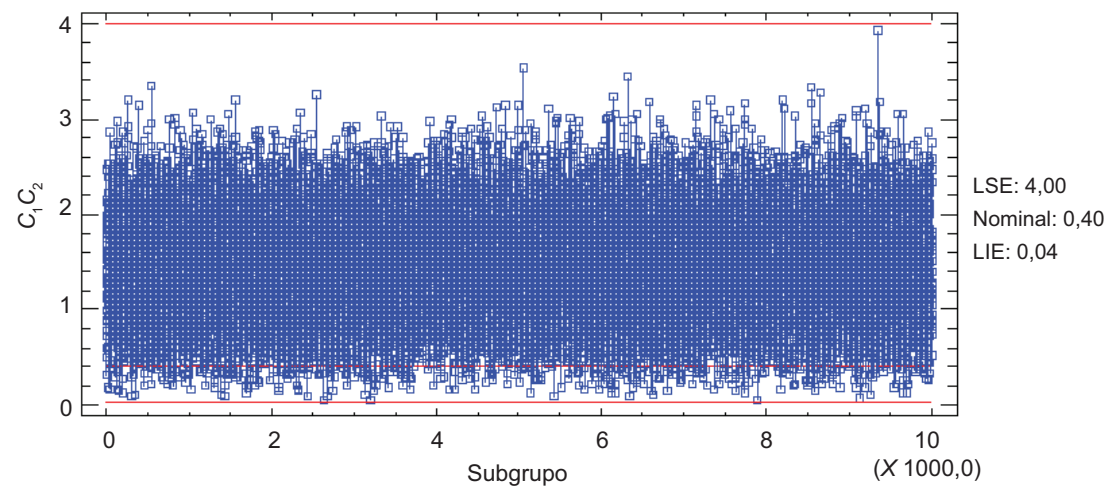

Figura 2. Gráficos de tolerancia para las variables $C_{1}, C_{2}$. Caso variables no correlacionadas. $n=10 ; 20 ; 50 ; 100 ; 1000$ y 10000

Elaboración propia 


\section{DISCUSIÓN}

Para esta investigación de variables no normales, las variables con correlación y sin correlación de los casos estudiados pueden modelarse a través de una distribución de Weibull multivariada. Con respecto a las variables correlacionadas, para los casos de tamaños muestrales $n=10 ; 20 ; 50$ y 100, el índice CPME que se ha propuesto en este artículo coincide con el índice de Cuamea y Rodríguez en cuanto a lo señalado acerca de que el proceso no es capaz; pero no hay coincidencia para las muestras $\quad 1000$ y $n=10000$. Sin embargo, el índice de Taam establece que el proceso es capaz para todos los casos muestrales, y el índice de Statgraphics coincide con el índice de esta investigación en cuanto a que el proceso no es capaz para todos los casos muéstrales. La figura 1 indica que el proceso no es capaz para los diferentes casos muestrales y corrobora la información indicada por el índice CPME y el índice obtenido por Statgraphics. Para el caso de variables no correlacionadas, el índice CPME muestra que para los tamaños muéstrales $n=10 ; 20 ; 50 ; 100$ y 1000, el proceso no es capaz, hecho que coincide con los índices de Cuamea y Rodríguez, Taam y Statgraphics; sin embargo, para el caso de $n=10000$, solo el índice $C P M E$ establece que el proceso es capaz, hecho que no expresan los índices de Cuamea y Rodríguez, Taam y Statgraphics. Además, el índice que aquí se plantea es respaldado por las gráficas de tolerancia de la figura 2. Las ventajas que presenta el índice CPME es que puede ser usado en ambos casos de variables con correlación (situación más compleja, ya que los otros ICPM fueron distorsionados por este caso) y sin correlación, no requiere de cálculos complejos y es fácil de calcular e interpretar.

\section{REFERENCIAS}

Ahmad, S., Abdollahian, M., Zeephongsekul, P., y Abbasi, B. (2009). Multivariate Non Normal Process Capability Analysis. International Journal of Advanced Manufacturing Technology, 4(7), 757-765.

Bothe, D. R. (1999). Composite Capability Index for Multiple Product Characteristics. Quality Engineering, 12(2), 253-258.

Box, G., y Cox, D. R. (1964). An Analysis of Transformation. Journal of the Royal Statistical Society: Series B, (26), 211-243. 
Castagliola, P., y Castellanos, J. V. G. (2005). Capability Indices Dedicated to the Two Quality Characteristics Case. Quality Technology and Quantitative Management, 2(2), 201-220.

Castagliola, P., y Castellanos, J. V. G. (2008). Process Capability Indices Dedicated to Bivariate Non Normal Distributions. Journal of Quality in Maintenance Engineering, 14(1), 87-101.

Choi, I. S., y Bai, D. S. (1996). Process Capability Index for Skewed Populations. En Proceedings of $20^{\text {th }}$ International Conference on Computers and Industrial Engineering (pp. 1211-1214). Kyongju.

Chou, Y., Polansky, A., y Mason, R. (1998). Transforming Non-Normal Data to Normality in Statistical Process Control. Journal of Quality Tecnology, (30), 133-141.

Cuamea, G., y Anaya, C. (2009). Evaluación de la calidad en productos o procesos con múltiples características de calidad correlacionadas. Epistemus, (6), 12-16.

Cuamea, G., y Rodríguez, M. (2014). Propuesta para evaluar la capacidad de procesos de manufactura multivariados. Revista Ingeniería Industrial, (2), 35-47.

Foster, E. J., Barton, R. R., y Gautam, N. (2005). The ProcessOriented Multivariate Capability Index. International Journal of Production Research, 43(10), 2135-2148.

Goethals, P. L., y Cho, B. R. (2011). The Development of a TargetFocused Process Capability Index with Multiple Characteristics. Quality and Reliability Engineering International, 27(3), 297311.

González, I., y Sánchez, I. (2009). Capability Indices and Nonconforming Proportion in Univariate and Multivariate Process. The International Journal of Advanced Manufacturing Technology, 44(9), 1036-1050.

Hubele, N., Shahiari, H., y Cheng, C. (1991). A Bivariate Capability Vector. En J. B. Keats y D. C. Montgomery (Eds.), Statistics and Design in Process Control: Keeping Pace with Automated Manufacturing (pp. 229-310). Nueva York: Marcel Dekker.

Johnson, N. L. (1949). System of Frequency Curves Generated by Methods of Translation. Biometrika, (36), 149-176. 
Kane, V. E. (1986). Process Capability Indices. Journal of Quality Technology, 34(1), 1-19.

Kotz, S., y Johnson, N. L. (2002). Process Capability Indices - a Review, 1992-2000. Journal of Quality Technology, 34(1), 2-19.

Liu, P. H., y Chen, F. L. (2006). Process Capability Analysis of Nonnormal Process Data Using the Burr XII Distribution. International Journal of Advanced Manufacturing Technology, 27(9), 975-984.

Montgomery, D. (2009). Statistical Quality Control. A Modern Introduction (6. ${ }^{\mathrm{a}}$ ed.). Nueva York: John Wiley and Sons.

Pal, S. (2005). Evaluation of Nonnormal Process Capability Indices Using Generalized Lambda Distribution. Quality Engineering, 17(1), 77-85.

Pan, J. N., y Lee, C. Y. (2010). New Capability Indices for Evaluating the Performance of Multivariate Manufacturing Processes. Quality and Reliability Engineering International, 26(1), 3-15.

Polansky, A. M., Chou, Y. M., y Mason, R. L. (1998). Estimating Process Capability Indices for a Truncated Distribution. Quality Engineering, 11(2), 257-265.

Shahriari, H., y Abdollahzadeh, M. (2009). A New Multivariate Process Capability Vector. Quality Engineering, 21(3), 290-299.

Shahriari, H., y Lawrence, F. P. (1995). A Multivariate Process Capability Vector. En Proceedings of the $4^{\text {th }}$ Industrial Engineering Research Conference (304-309). Nashville.

Shinde, R. L., y Khadse, K. G. (2009). Multivariate Process Capability using Principal Component Analysis. Quality and Reliability Engineering International, 25(1), 69-77.

Somerville, S., y Montgomery, D. (1996). Process Capability Indices and Non-Normal Distributions. Quality Engieneering, (19), 305-316.

Taam, W., y Liddy, J. W. (1993). A Note of Multivariate Capability Indices. Journal of Applied Statistics, 20(3), 339-351.

Tang, L. C., y Than, S. E. (1999). Computing Process Capability Indices for Non-Normal Data: a Review And Comparative Study. Quality and Reliability Engineering International (15), 339-353. 
Valdiviezo, M., y Fermín, J. (2010). Estudio comparativo de índices de capacidad de proceso con variables distribuidas no normales. Industrial Data, 13(2), 56-65.

Wang, F. K., y Chen, J. C. (1998). Capability Index Using Principal Component Analysis. Quality Engineering, 11(1), 21-27.

Wang, F. K. (2006). Quality Evaluations of a Manufactured Product with Multiple Characteristics. Quality and Reliability Engineering International, 22(2), 225-236.

Wang, F. K., y Du, T. C. (2000) Using Principal Component Analysis in Process Performance for Multivariate Data. Omega, 28(2), 185-194.

Wang, F. K., Hubele, N. F., Lawrence, F. P., Miskulin, J. D., y Shahriari, H. (2000). Comparison for Three Multivariate Process Capability Indices. Journal of Quality Technology, 32(3), 263-275.

Yeh, A., y Chen, H. (2001). A Nonparametric Multivariate Process Capability Index. International Journal of Modelling and Simulation, 21(3), 218-223. 
